\title{
Exact Solutions of the Vakhnenko-Parkes Equation with Complex Method
}

\author{
Yongyi Gu, Wenjun Yuan, Najva Aminakbari, and Qinghua Jiang \\ School of Mathematics and Information Science, Guangzhou University, Guangzhou 510006, China \\ Correspondence should be addressed to Wenjun Yuan; wjyuan1957@126.com
}

Received 11 May 2017; Accepted 26 September 2017; Published 9 November 2017

Academic Editor: Hua Su

Copyright (c) 2017 Yongyi Gu et al. This is an open access article distributed under the Creative Commons Attribution License, which permits unrestricted use, distribution, and reproduction in any medium, provided the original work is properly cited.

We derive exact solutions to the Vakhnenko-Parkes equation by means of the complex method, and then we illustrate our main results by some computer simulations. We can apply the idea of this study to related nonlinear differential equation.

\section{Introduction and Main Results}

Nonlinear differential equations are widely used as models to describe many important dynamical systems in various fields of science, especially in nonlinear optics, plasma physics, solid state physics, and fluid mechanics. It has aroused extensive interest in the study of nonlinear differential equations [1-15].

In 1992, Vakhnenko [16] first presented the nonlinear differential equation

$$
\frac{\partial}{\partial x}\left(u \frac{\partial}{\partial x}+\frac{\partial}{\partial t}\right)+u=0
$$

and obtained solitary wave solutions to (1). The equation above gives a description of high-frequency waves in the relaxation medium [17].

In 1998, Vakhnenko and Parkes [18] found the soliton solution to the transformed form of (1) as follows:

$$
u u_{x x t}-u_{x} u_{x t}+u^{2} u_{t}=0
$$

Hereafter (2) is called the Vakhnenko-Parkes equation (VPE). In recent years, many powerful methods for constructing the solutions of VPE have been used, for instance, the HirotaBacklund transformations method [19], the inverse scattering method [20, 21], the exp-function method [22], and the $\left(G^{\prime} / G\right)$-expansion method [23]. In this article, we would like to use the complex method [24-26] to obtain traveling wave solutions of VPE.
Substituting traveling wave transform

$$
u(x, t)=w(z), \quad z=v x-\delta t,
$$

into Vakhnenko-Parkes (2), we get

$$
v^{2} w w^{\prime \prime \prime}-v^{2} w^{\prime} w^{\prime \prime}+w^{2} w^{\prime}=0
$$

Integrating (4) yields

$$
3 v^{2} w w^{\prime \prime}-3 v^{2}\left(w^{\prime}\right)^{2}+w^{3}-\lambda=0
$$

where $\nu$ and $\lambda$ are constants

If a meromorphic function $g$ is a rational function of $z$, or a rational function of $e^{\mu z}, \mu \in \mathbb{C}$, or an elliptic function, then we say that $g$ belongs to the class $W$ [27].

Theorem 1. If $v \neq 0$, then the meromorphic solutions $w$ of (5) belong to the class W. In addition, (5) has the following classes of solutions.

(I) The Rational Function Solutions

$$
W_{r}(z)=\frac{-6 v^{2}}{\left(z-z_{0}\right)^{2}}
$$

where $\lambda=0, z_{0} \in \mathbb{C}$.

(II) The Simply Periodic Solutions

$$
W_{s}(z)=-\frac{3 \nu^{2} \mu^{2}}{2} \operatorname{coth}^{2} \frac{\mu\left(z-z_{0}\right)}{2}+\frac{\left(3 \mu^{2}-1\right) \nu^{2}}{2},
$$

where $\lambda=v^{6} / 8, z_{0} \in \mathbb{C}$. 
(III) The Elliptic Function Solutions

$$
W_{d}(z)=-6 v^{2}\left(-\wp(z)+\frac{1}{4}\left(\frac{\wp^{\prime}(z)+D}{\wp(z)-C}\right)^{2}\right)+6 \nu^{2} C,
$$

where $D^{2}=4 C^{3}-c_{3}, c_{2}=0$, and $c_{3}=-\lambda / 108 v^{6}$.

\section{Preliminaries}

At first, we give some notations and definitions, and then we introduce some lemmas.

Let $m \in \mathbb{N}^{*}:=\{1,2,3, \ldots\}, r_{j} \in \mathbb{N}=\mathbb{N}^{*} \cup\{0\}, r=$ $\left(r_{0}, r_{1}, \ldots, r_{m}\right), j=0,1, \ldots, m$, and

$$
\begin{aligned}
& K_{r} {[w](z) } \\
& \quad:=[w(z)]^{r_{0}}\left[w^{\prime}(z)\right]^{r_{1}}\left[w^{\prime \prime}(z)\right]^{r_{2}} \cdots\left[w^{(m)}(z)\right]^{r_{m}} ;
\end{aligned}
$$

then $d(r):=\sum_{j=0}^{m} r_{j}$ is the degree of $K_{r}[w]$. Let the differential polynomial be defined by

$$
F\left(w, w^{\prime}, \ldots, w^{(m)}\right):=\sum_{r \in J} a_{r} K_{r}[w],
$$

where $J$ is a finite index set and $a_{r}$ are constants; then $\operatorname{deg} F\left(w, w^{\prime}, \ldots, w^{(m)}\right):=\max _{r \in J}\{d(r)\}$ is the degree of $F\left(w, w^{\prime}, \ldots, w^{(m)}\right)$.

Consider the following differential equation:

$$
F\left(w, w^{\prime}, \ldots, w^{(m)}\right)=c w^{n}+d,
$$

where $c \neq 0, d$ are constants, $n \in \mathbb{N}^{*}$.

Let $p, q \in \mathbb{N}^{*}$, and assume that the meromorphic solutions $w$ of (11) have at least one pole. If (11) has exactly $p$ distinct meromorphic solutions, and their multiplicity of the pole at $z=0$ is $q$, then (11) is said to satisfy the $\langle p, q\rangle$ condition. It is not easy to verify that the $\langle p, q\rangle$ condition of (11) holds, so we need the weak $\langle p, q\rangle$ condition as follows.

By substituting the Laurent series

$$
w(z)=\sum_{\tau=-q}^{\infty} \beta_{\tau} z^{\tau}, \quad \beta_{-q} \neq 0, q>0,
$$

into (11), we determine exactly $p$ different Laurent singular parts:

$$
\sum_{\tau=-q}^{-1} \beta_{\tau} z^{\tau}
$$

then (11) is said to satisfy the weak $\langle p, q\rangle$ condition.

Given two complex numbers $l_{1}, l_{2}$ such that $\operatorname{Im}\left(l_{1} / l_{2}\right)>$ 0 , and let $L$ be the discrete subset $L\left[2 l_{1}, 2 l_{2}\right]:=\{l \mid l=$ $\left.2 a l_{1}+2 b l_{2}, a, b \in \mathbb{Z}\right\}$, and $L$ is isomorphic to $\mathbb{Z} \times \mathbb{Z}$. Let the discriminant $\Delta=\Delta\left(b_{1}, b_{2}\right):=b_{1}^{3}-27 b_{2}^{2}$ and

$$
H_{n}=H_{n}(L):=\sum_{l \in L \backslash\{0\}} \frac{1}{l^{n}} .
$$

A meromorphic function $\wp(z):=\wp\left(z, c_{2}, c_{3}\right)$ with double periods $2 l_{1}, 2 l_{2}$, which satisfies the equation

$$
\left(\wp^{\prime}(z)\right)^{2}=4 \wp(z)^{3}-c_{2} \wp(z)-c_{3},
$$

where $c_{2}=60 H_{4}, c_{3}=140 H_{6}$, and $\Delta\left(c_{2}, c_{3}\right) \neq 0$, is called the Weierstrass elliptic function.

In 2009, Eremenko et al. [28] studied the $m$-order BriotBouquet equation (BBEq)

$$
F\left(w, w^{(m)}\right)=\sum_{j=0}^{n} F_{j}(w)\left(w^{(m)}\right)^{j}=0,
$$

where $F_{j}(w)$ are constant coefficients polynomials, $m \in \mathbb{N}^{*}$. For the $m$-order BBEq, we have the following lemma.

Lemma 2 (see $[26,29,30]$ ). Let $m, n, p, s \in \mathbb{N}^{*}$, $\operatorname{deg} F\left(w, w^{(m)}\right)<n$. If a $m$-order BBEq

$$
F\left(w, w^{(m)}\right)=c w^{n}+d
$$

satisfies the weak $\langle p, q\rangle$ condition, then the meromorphic solutions $w$ belong to the class $W$. Suppose that for some values of parameters such solution $w$ exists; then other meromorphic solutions form a one-parametric family $\left(z-z_{0}\right), z_{0} \in \mathbb{C}$. Furthermore, each elliptic solution with pole at $z=0$ can be written as

$$
\begin{aligned}
& w(z) \\
& =\sum_{i=1}^{s-1} \sum_{j=2}^{q} \frac{(-1)^{j} \beta_{-i j}}{(j-1) !} \frac{d^{j-2}}{d z^{j-2}}\left(\frac{1}{4}\left[\frac{\wp^{\prime}(z)+C_{i}}{\wp(z)-D_{i}}\right]^{2}-\wp(z)\right) \\
& \quad+\sum_{i=1}^{s-1} \frac{\beta_{-i 1}}{2} \frac{\wp^{\prime}(z)+C_{i}}{\wp(z)-D_{i}} \\
& \quad+\sum_{j=2}^{q} \frac{(-1)^{j} \beta_{-s j}}{(j-1) !} \frac{d^{j-2}}{d z^{j-2}} \wp(z)+\beta_{0},
\end{aligned}
$$

where $\beta_{-i j}$ are determined by (12), $\sum_{i=1}^{s} \beta_{-i 1}=0$, and $C_{i}^{2}=$ $4 D_{i}^{3}-c_{2} D_{i}-c_{3}$.

Each rational function solution has $s(\leq p)$ distinct poles of multiplicity $q$ and is expressed as

$$
R(z)=\sum_{i=1}^{s} \sum_{j=1}^{q} \frac{\beta_{i j}}{\left(z-z_{i}\right)^{j}}+\beta_{0} .
$$

Each simply periodic solution has $s(\leq p)$ distinct poles of multiplicity $q$ and is expressed as

$$
R(\eta)=\sum_{i=1}^{s} \sum_{j=1}^{q} \frac{\beta_{i j}}{\left(\eta-\eta_{i}\right)^{j}}+\beta_{0},
$$

which is a rational function of $\eta=e^{\mu z}(\mu \in \mathbb{C})$. 
Lemma 3 (see [27, 30]). Weierstrass elliptic functions $\wp(z):=$ $\wp\left(z, c_{2}, c_{3}\right)$ have an addition formula as below:

$$
\wp\left(z-z_{0}\right)=\frac{1}{4}\left[\frac{\wp^{\prime}(z)+\wp^{\prime}\left(z_{0}\right)}{\wp(z)-\wp\left(z_{0}\right)}\right]^{2}-\wp\left(z_{0}\right)-\wp(z) .
$$

When $c_{2}=c_{3}=0$, Weierstrass elliptic functions can be degenerated to rational functions according to

$$
\wp(z, 0,0)=\frac{1}{z^{2}} \text {. }
$$

When $\Delta\left(c_{2}, c_{3}\right)=0$, Weierstrass elliptic functions can be degenerated to simple periodic functions according to

$$
\wp\left(z, 3 d^{2},-d^{3}\right)=2 d-\frac{3 d}{2} \operatorname{coth}^{2} \sqrt{\frac{3 d}{2}} z .
$$

\section{Proof of Theorem 1}

Substituting (12) into (5) we obtain $p=1, q=2, \beta_{-2}=$ $-6 v^{2}, \beta_{-1}=0, \beta_{0}$ is an arbitrary constant, $\beta_{1}=0, \beta_{2}=$ $-\beta_{0}^{2} / 10 v^{2}$, and $\beta_{3}=0$.

Multiplying (5) by $w^{\prime} / w^{3}$, we get

$$
\frac{3 v^{2} w^{\prime} w^{\prime \prime}}{w^{2}}-\frac{3 v^{2}\left(w^{\prime}\right)^{3}}{w^{3}}+w^{\prime}-\frac{\lambda w^{\prime}}{w^{3}}=0 .
$$

Integrating (24) yields

$$
3 v^{2}\left(w^{\prime}\right)^{2}+2 w^{3}+2 \gamma w^{2}-\lambda=0,
$$

where $\nu$ is an arbitrary constant and $\gamma$ is the integrable constant.

Therefore, (25) is a first-order BBEq and satisfies the weak $\langle 1,2\rangle$ condition. Hence, by Lemma 2 , the meromorphic solutions of (25) $w \in W$. It means that the meromorphic solutions of (5) $w \in W$. The forms of the meromorphic solutions to (5) will be given in the following.

By (19), we infer that the indeterminate rational solutions of (5) are

$$
R_{1}(z)=\frac{\beta_{11}}{z^{2}}+\frac{\beta_{12}}{z}+\beta_{10}
$$

with pole at $z=0$.

Substituting $R_{1}(z)$ into (5), we have

$$
\begin{aligned}
& \frac{\beta_{11}^{2}\left(6 v^{2}+\beta_{11}\right)}{z^{6}}+\frac{3 \beta_{11} \beta_{12}\left(4 v^{2}+\beta_{11}\right)}{z^{5}} \\
& +\frac{3\left(6 \beta_{11} \beta_{10} \nu^{2}+\beta_{12}^{2} \nu^{2}+\beta_{11}^{2} \beta_{10}+\beta_{11} \beta_{12}^{2}\right)}{z^{4}} \\
& +\frac{\beta_{12}\left(6 \beta_{10} v^{2}+6 \beta_{11} \beta_{10}+\beta_{12}^{2}\right)}{z^{3}} \\
& \quad+\frac{3 \beta_{10}\left(\beta_{11} \beta_{10}+\beta_{12}^{2}\right)}{z^{2}}+\frac{3 \beta_{12} \beta_{10}^{2}}{z}+\beta_{10}^{3}+\lambda \\
& =0 ;
\end{aligned}
$$

then we get $\beta_{11}=-6 v^{2}, \beta_{12}=\beta_{10}=0$.
Therefore, we can determine that

$$
R_{1}(z)=\frac{-6 v^{2}}{z^{2}}
$$

where $\lambda=0$.

So the rational solutions of (5) are

$$
W_{r}(z)=\frac{-6 v^{2}}{\left(z-z_{0}\right)^{2}}
$$

where $\lambda=0, z_{0} \in \mathbb{C}$.

To obtain simply periodic solutions, let $\eta=e^{\mu z}$, and substitute $w=R(\eta)$ into (5); then

$$
3 v^{2} \mu^{2} R\left(\eta R^{\prime}+\eta^{2} R^{\prime \prime}\right)-3 v^{2} \mu^{2}\left(R^{\prime} \eta\right)^{2}+R^{3}+\lambda=0 .
$$

Substituting

$$
R_{2}(z)=\frac{\beta_{21}}{(\eta-1)^{2}}+\frac{\beta_{22}}{(\eta-1)}+\beta_{20}
$$

into (30), we obtain that

$$
R_{2}(z)=-\frac{6 \nu^{2} \mu^{2}}{(\eta-1)^{2}}-\frac{6 v^{2} \mu^{2}}{(\eta-1)}-\frac{\nu^{2}}{2}
$$

where $\lambda=\nu^{6} / 8$.

Substituting $\eta=e^{\mu z}$ into (32), we can get simply periodic solutions to (5) with pole at $z=0$

$$
\begin{aligned}
W_{s 0}(z) & =-\frac{6 v^{2} \mu^{2}}{\left(e^{\mu z}-1\right)^{2}}-\frac{6 v^{2} \mu^{2}}{\left(e^{\mu z}-1\right)}-\frac{\nu^{2}}{2} \\
& =-6 v^{2} \mu^{2} \frac{e^{\mu z}}{\left(e^{\mu z}-1\right)^{2}}-\frac{v^{2}}{2} \\
& =-\frac{3 v^{2} \mu^{2}}{2} \operatorname{coth}^{2} \frac{\mu z}{2}+\frac{\left(3 \mu^{2}-1\right) \nu^{2}}{2},
\end{aligned}
$$

where $\lambda=v^{6} / 8$

So simply periodic solutions of (5) are

$$
W_{s}(z)=-\frac{3 v^{2} \mu^{2}}{2} \operatorname{coth}^{2} \frac{\mu\left(z-z_{0}\right)}{2}+\frac{\left(3 \mu^{2}-1\right) \nu^{2}}{2},
$$

where $\lambda=v^{6} / 8, z_{0} \in \mathbb{C}$.

From (18) of Lemma 2, we can express the elliptic solutions of (5) as

$$
w_{d 0}(z)=\beta_{-2 \wp} \wp(z)+\beta_{30}
$$

with pole at $z=0$.

Putting $w_{d 0}(z)$ into (5), we obtain that

$$
w_{d 0}(z)=-6 v^{2} \wp(z),
$$

where $c_{2}=0$ and $c_{3}=-\lambda / 108 v^{6}$. 


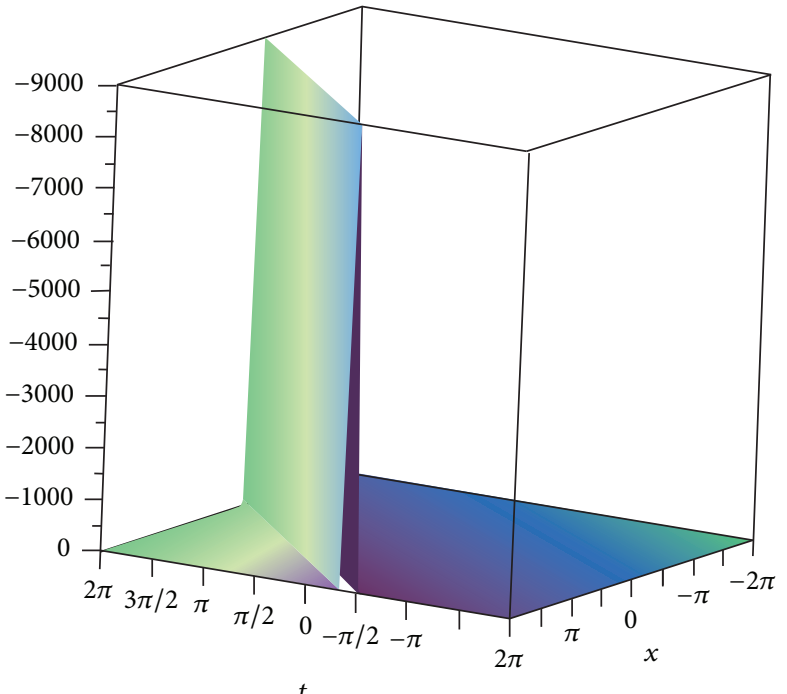

(a)

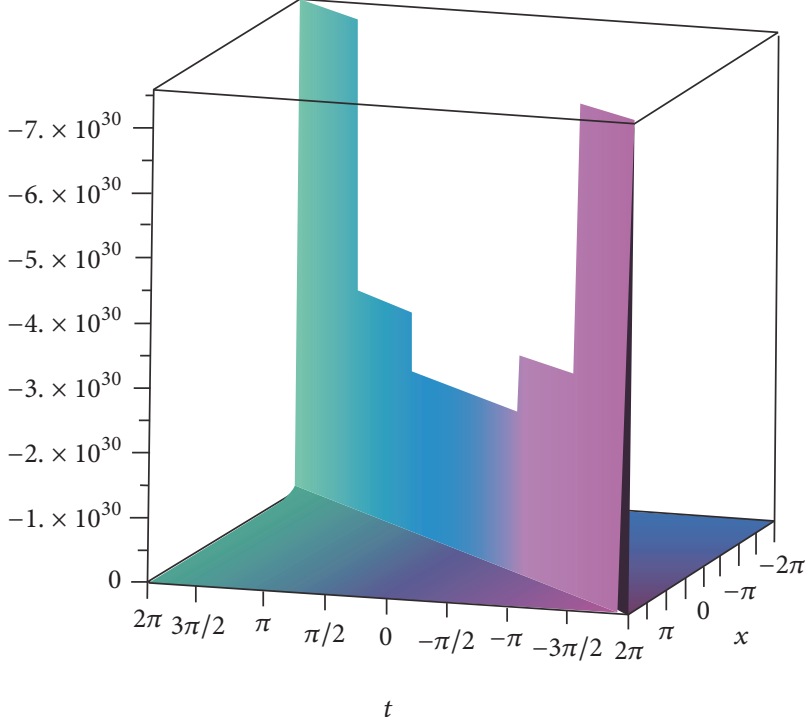

(b)

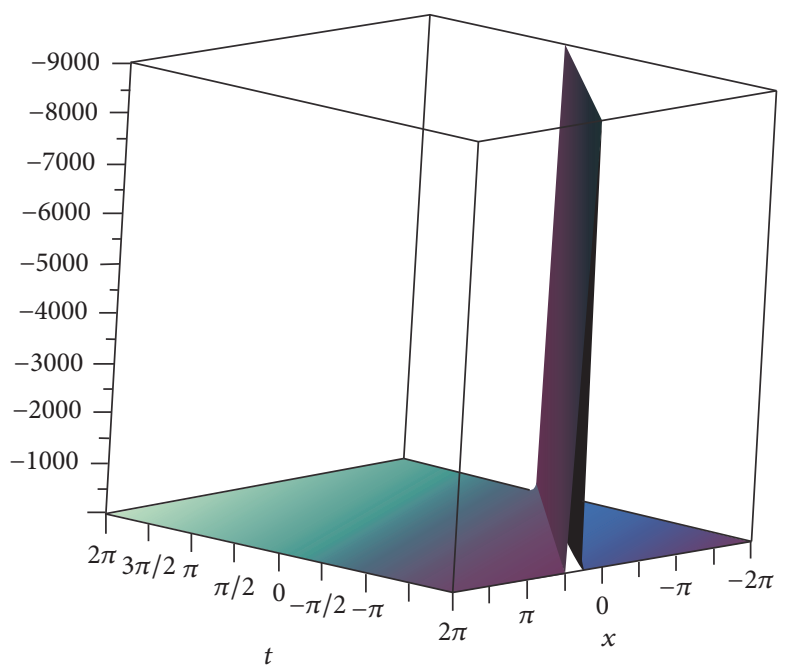

(c)

FIGURE 1: The solution of the Vakhnenko-Parkes equation corresponding to $W_{r}(z)$, (a) $z_{0}=5$, (b) $z_{0}=0$, and (c) $z_{0}=-5$.

Therefore, the elliptic solutions of (5) are

$$
W_{d}(z)=-6 v^{2} \wp\left(z-z_{0}\right)
$$

where $z_{0} \in \mathbb{C}$.

Applying the addition formula, we can rewrite it as

$$
\begin{aligned}
W_{d}(z)= & -6 \nu^{2}\left(-\wp(z)+\frac{1}{4}\left(\frac{\wp^{\prime}(z)+D}{\wp(z)-C}\right)^{2}\right) \\
& +6 \nu^{2} C,
\end{aligned}
$$

where $D^{2}=4 C^{3}-c_{3}, c_{2}=0$, and $c_{3}=-\lambda / 108 \nu^{6}$.

\section{Computer Simulations}

In this section, we illustrate our main results by some computer simulations. We carry out further analysis to the properties of simply periodic solutions $W_{s}(z)$ and the rational solutions $W_{r}(z)$ as in Figures 1 and 2.

(1) For $W_{s}(z)$, take $\nu=1, \delta=1$, and $\mu=\sqrt{2} / 2$.

(2) For $W_{r}(z)$, take $v=1$ and $\delta=1$.

\section{Conclusions}

Employing the complex method, we can easily find exact solutions to some nonlinear differential equation. By this method, we get the meromorphic exact solutions of VPE, and then we obtain the traveling wave solutions to VPE. In $W_{r}(z)$ of our solutions, let $z=k x+w t$ and $z_{0}=-c_{1} / c_{2}$; then it will be equivalent to Eq. (20) in [23]. Simply periodic solutions $W_{s}(z)$ are new and cannot be degenerated through elliptic function solutions. Our results demonstrate that the complex method 


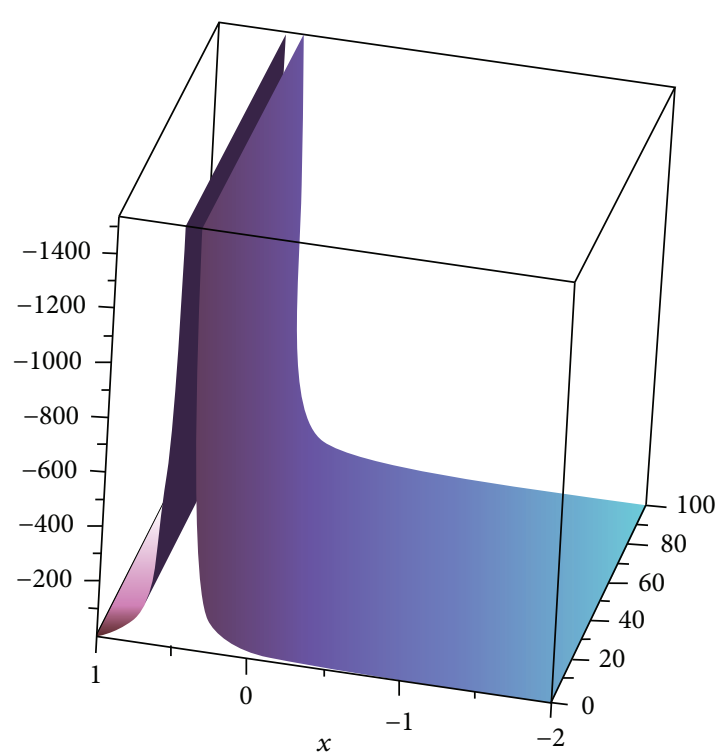

(a)

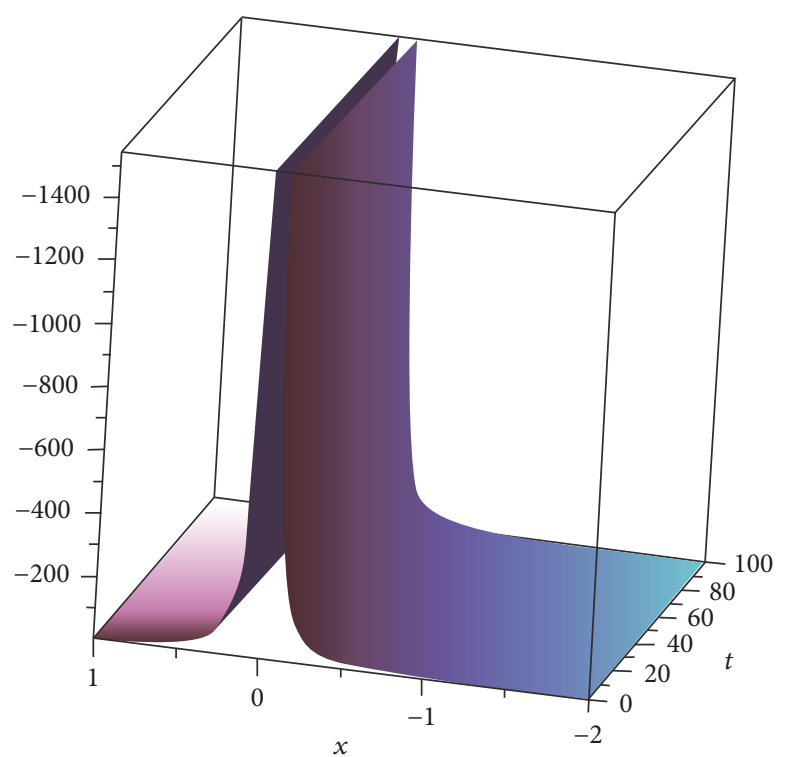

(b)

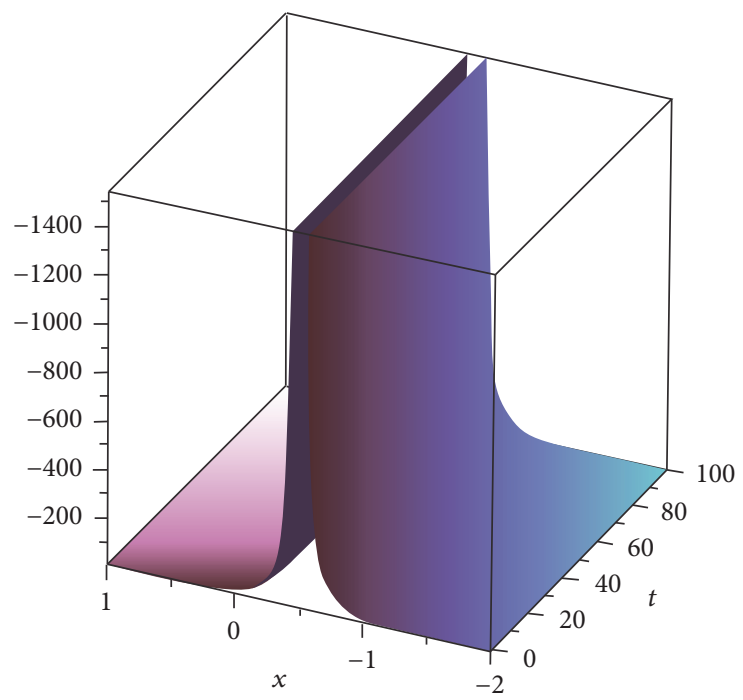

(c)

FIGURE 2: The solution of the Vakhnenko-Parkes equation corresponding to $W_{r}(z)$, (a) $z_{0}=0.5$, (b) $z_{0}=0$, and (c) $z_{0}=-0.5$.

is more simpler, and we can apply the idea of this study to related nonlinear evolution equation.

\section{Conflicts of Interest}

The authors declare that there are no conflicts of interest regarding the publication of this paper.

\section{Authors' Contributions}

Yongyi Gu and Wenjun Yuan carried out the design of this paper and performed the analysis. Najva Aminakbari and Qinghua Jiang participated in the calculations and computer simulations. All authors typed, read, and approved the final manuscript.

\section{Acknowledgments}

This work was supported by the NSF of China (11271090), the NSF of Guangdong Province (2016A030310257), Young Talents Innovation Project of Guangdong Province (2015KQNCX116), and Joint PHD Program of Guangzhou University and Curtin University.

\section{References}

[1] A. Mao and S. Luan, "Periodic solutions of an infinitedimensional Hamiltonian system," Applied Mathematics and Computation, vol. 201, no. 1-2, pp. 800-804, 2008.

[2] F. Li and Y. Bai, "Uniform decay rates for nonlinear viscoelastic Marguerre-von Kármán equations," Journal of Mathematical Analysis and Applications, vol. 351, no. 2, pp. 522-535, 2009. 
[3] F. Li, "Limit behavior of the solution to nonlinear viscoelastic Marguerre-von Kármán shallow shell system," Journal of Differential Equations, vol. 249, no. 6, pp. 1241-1257, 2010.

[4] Y. Guo, "Nontrivial solutions for boundary-value problems of nonlinear fractional differential equations," Bulletin of the Korean Mathematical Society, vol. 47, no. 1, pp. 81-87, 2010.

[5] Y. Guo, "Solvability of boundary-value problems for nonlinear fractional differential equations," Ukrainian Mathematical Journal, vol. 62, no. 9, pp. 1409-1419, 2011.

[6] J. Jiang, L. Liu, and Y. Wu, "Multiple positive solutions of singular fractional differential system involving Stieltjes integral conditions," Electronic Journal of Qualitative Theory of Differential Equations, vol. 2012, no. 43, 18 pages, 2012.

[7] J. Jiang, L. Liu, and Y. Wu, "Positive solutions for p-laplacian fourth-order differential system with integral boundary conditions," Discrete Dynamics in Nature and Society, vol. 2012, Article ID 293734, 19 pages, 2012.

[8] J. Jiang, L. Liu, and Y. Wu, "Positive solutions to singular fractional differential system with coupled boundary conditions," Communications in Nonlinear Science and Numerical Simulation, vol. 18, no. 11, pp. 3061-3074, 2013.

[9] J. Jiang, L. Liu, and Y. Wu, "Symmetric positive solutions to singular system with multi-point coupled boundary conditions," Applied Mathematics and Computation, vol. 220, pp. 536-548, 2013.

[10] L. Liu, X. Hao, and Y. Wu, "Positive solutions for singular second order differential equations with integral boundary conditions," Mathematical and Computer Modelling, vol. 57, no. 3-4, pp. 836847, 2013.

[11] L. S. Liu, J. Q. Jiang, and Y. H. Wu, “The unique solution of a class of sum mixed monotone operator equations and its application to fractional boundary value problems," Journal of Nonlinear Science and Applications, vol. 9, no. 5, pp. 2943-2958, 2016.

[12] J. Jiang and L. Liu, "Existence of solutions for a sequential fractional differential system with coupled boundary conditions," Boundary Value Problems, vol. 2016, no. 159, 15 pages, 2016.

[13] F. Li and Q. Gao, "Blow-up of solution for a nonlinear Petrovsky type equation with memory," Applied Mathematics and Computation, vol. 274, pp. 383-392, 2016.

[14] K. M. Zhang, "On a sign-changing solution for some fractional differential equations," Boundary Value Problems, vol. 2017, no. 59, 8 pages, 2017.

[15] J. Ding, J. Wang, and Z. Ye, "Growth of meromorphic solutions to some complex functional equations," Computational Methods and Function Theory, vol. 16, no. 3, pp. 489-502, 2016.

[16] V. A. Vakhnenko, "Solitons in a nonlinear model medium," Journal of Physics A: Mathematical and General, vol. 25, no. 15, pp. 4181-4187, 1992.

[17] V. O. Vakhnenko, "High-frequency soliton-like waves in a relaxing medium," Journal of Mathematical Physics, vol. 40, no. 4, pp. 2011-2020, 1999.

[18] V. O. Vakhnenko and E. J. Parkes, "The two loop soliton solution of the Vakhnenko equation," Nonlinearity, vol. 11, no. 6, pp. 1457-1464, 1998.

[19] A. J. Morrison, E. J. Parkes, and V. O. Vakhnenko, “The N loop soliton solution of the Vakhnenko equation," Nonlinearity, vol. 12, no. 5, pp. 1427-1437, 1999.

[20] V. O. Vakhnenko, E. J. Parkes, and A. V. Michtchenko, "The Vakhnenko equation from the viewpoint of the inverse scattering method for the KdV equation," International Journal of Differential Equations and Applications, vol. 1, no. 4, pp. 429449, 2000.
[21] V. O. Vakhnenko and E. J. Parkes, "The calculation of multisoliton solutions of the Vakhnenko equation by the inverse scattering method," Chaos, Solitons \& Fractals, vol. 13, no. 9, pp. 1819-1826, 2002.

[22] C. Köroglu and T. Özis, "A novel traveling wave solution for Ostrovsky equation using exp-function method," Computers and Mathematics with Applications, vol. 58, no. 11-12, pp. 21422146, 2009.

[23] R. Abazari, "Application of $\left(G^{\prime} / G\right)$-expansion method to travelling wave solutions of three nonlinear evolution equation," Computers \& Fluids. An International Journal, vol. 39, no. 10, pp. 1957-1963, 2010.

[24] W. Yuan, Y. Li, and J. Lin, "Meromorphic solutions of an auxiliary ordinary differential equation using complex method," Mathematical Methods in the Applied Sciences, vol. 36, no. 13, pp. 1776-1782, 2013.

[25] W. Yuan, F. Meng, Y. Huang, and Y. Wu, "All traveling wave exact solutions of the variant Boussinesq equations," Applied Mathematics and Computation, vol. 268, pp. 865-872, 2015.

[26] W. J. Yuan, Y. D. Shang, Y. Huang, and H. Wang, “The representation of meromorphic solutions to certain ordinary differential equations and its applications," Scientia Sinica Mathematica, vol. 43, no. 6, pp. 563-575, 2013.

[27] R. Conte and M. Musette, "Elliptic general analytic solutions," Studies in Applied Mathematics, vol. 123, no. 1, pp. 63-81, 2009.

[28] A. E. Eremenko, L. Liao, and T. W. Ng, "Meromorphic solutions of higher order Briot-Bouquet differential equations," Mathematical Proceedings of the Cambridge Philosophical Society, vol. 146, no. 1, pp. 197-206, 2009.

[29] N. A. Kudryashov, "Meromorphic solutions of nonlinear ordinary differential equations," Communications in Nonlinear Science and Numerical Simulation, vol. 15, no. 10, pp. 2778-2790, 2010.

[30] S. Lang, Elliptic Functions, Springer, New York, NY, USA, 2nd Edition edition, 1987. 


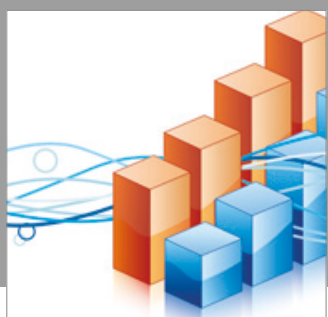

Advances in

Operations Research

vatersals

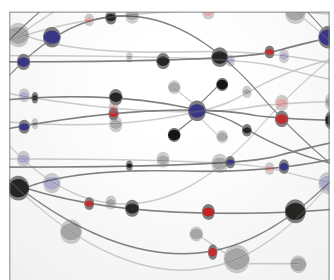

\section{The Scientific} World Journal
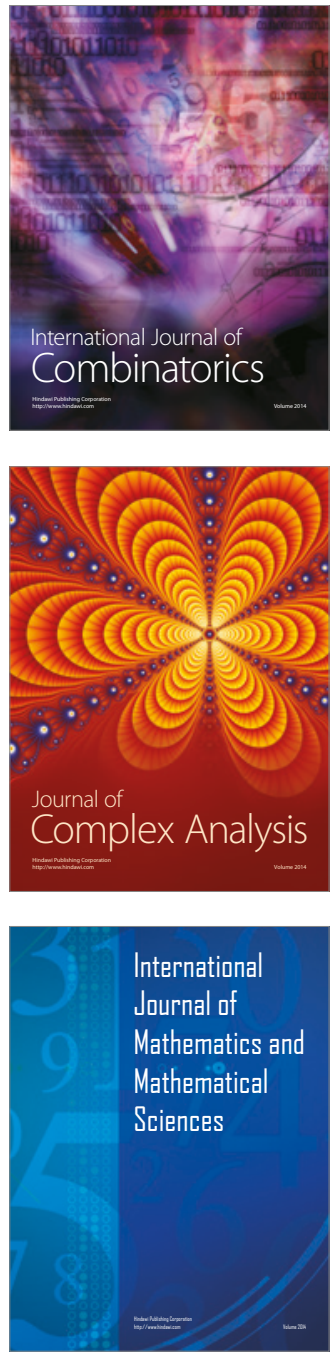
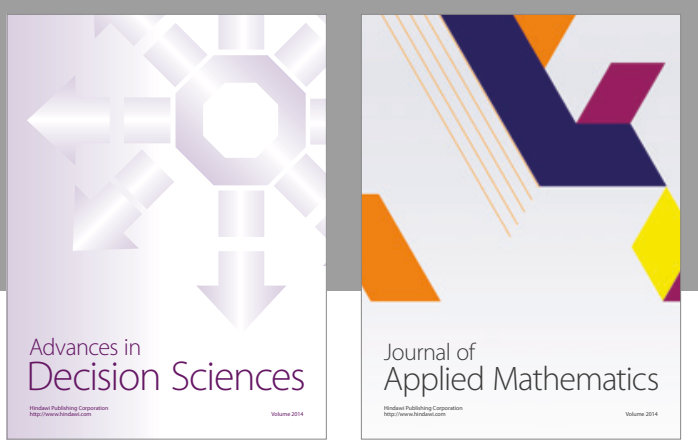

Algebra

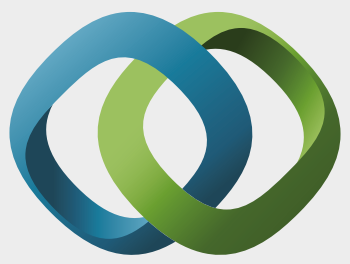

\section{Hindawi}

Submit your manuscripts at

https://www.hindawi.com
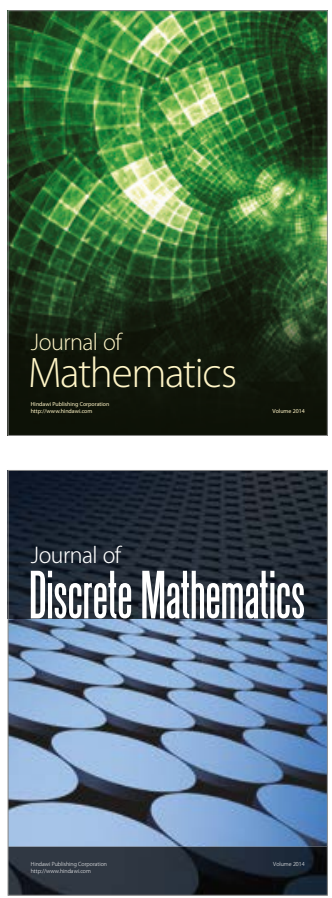

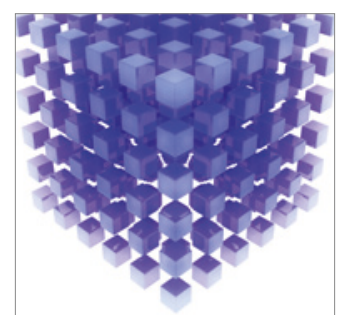

Mathematical Problems in Engineering
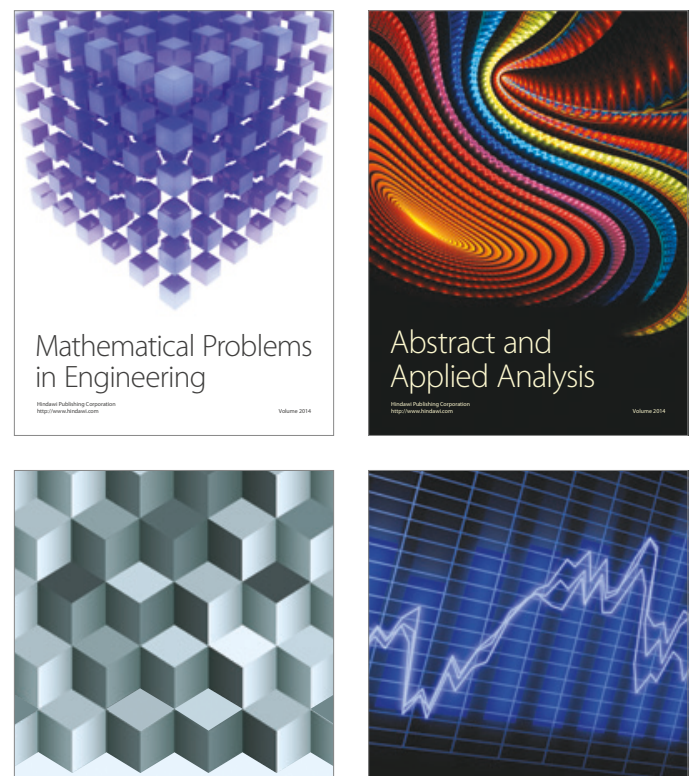

Journal of

Function Spaces

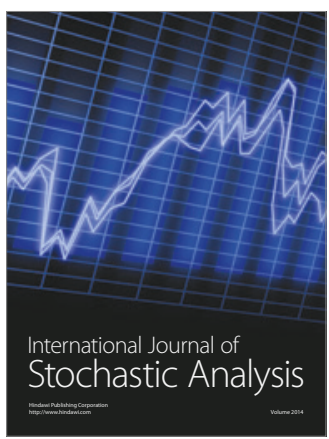

Probability and Statistics
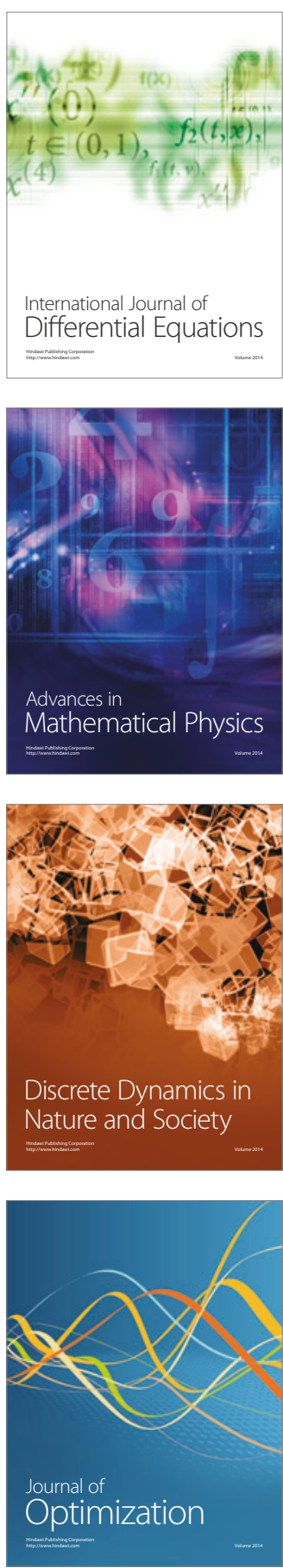